\title{
Resolving Conflicts between Personal and Normative Goals in Normative Agent Systems
}

\author{
Azhana Ahmad \\ Moamin Ahmed \\ College of Information \\ Technology \\ Universiti Tenaga Nasional \\ azhana@uniten.edu.my \\ moamin84@gmail.com
}

\author{
Mohd Zaliman Mohd Yusof \\ Mohd. Sharifuddin Ahmad \\ College of Information \\ Technology \\ Universiti Tenaga Nasional \\ \{zaliman, sharif\}@uniten.edu.my
}

\author{
Aida Mustapha \\ Faculty of Computer Science \& \\ Information Technology \\ Universiti Putra Malaysia \\ aida@fsktm.upm.edu.my
}

\begin{abstract}
In this paper, we present a framework for resolving conflicts between personal and normative goals in normative agent systems. The conflicts occur in the decision making process of time-constrained tasks of those goals. The agents observe the environment and perform the tasks based on their obligation to an authority, their desires, and intentions. They select and execute the tasks from a set of pre-compiled tasks based on their beliefs of the reward and penalty associated with the selected tasks. To resolve the conflicts within the constraint of the tasks' duration, we supplement the agents' normative capacity with two essential functions: Sacrifice and Diligence. The Sacrifice function enables an agent to reason and discard any tasks that have lower priorities to make way for accomplishment of the normative goal. The Diligence function enables an agent to increase its effort in accomplishing the normative goal in time-constrained situations. We simulate these situations and present the results.
\end{abstract}

Keywords: norms, software agents, intentional agent, multi-agent systems.

\section{Introduction}

Norms has become one of the areas of agent research to improve the predictability of agent society and to manifest orderly coordination and co-operation among the agents in the society. Norms are characterized by their prescriptiveness, sociality, and social pressure. Prescriptiveness in norms influences an agent on how to behave. Sociality is concerned with the achievement of some goal when more than one agent is involved. Since it is always expected that norms conflict with the personal interests of some agents, social pressure, which is a set of socially acceptable mechanisms to force agents to comply with norms are needed [4]. In a norm-based Belief-Desire-Intention (BDI) architecture, agents are built with mentalistic notions to recognize or deliberate their personal goals, while at the same time to fulfill their normative goals as imposed by an authority in a society. In this instance, violations could occur when agents attempt to achieve their personal goals, hence affecting their normative goal. Consequently, norms internalization [15] is an important issue to consider when agents regulate the norms without coercion and at the same time accomplish their personal goals. A typical scenario for such instance could be a student who needs to submit an assignment in four weeks time. In this case, the normative goal is the submission of the assignment. The student has other personal goals such as visiting a sick grandmother, meeting old friends, attending the class for other courses, preparing for the coming examination, and so forth. The student needs to prioritize all tasks so that the assignment submission will occur in one of the days before the deadline. Some tasks may have to be forfeited or sacrificed to make way for the normative goal. He/She may cancel the plan to meet old friends or may have to increase his/her diligence or the level of effort to complete and submit the assignment.

To resolve such conflicts, we introduce two new functions: Sacrifice and Diligence to the BDI architecture of the Obligation-Prohibition-Recommended-Neutrality-Disliked (OP-RND) framework [1]. The Sacrifice function enables an agent to reason and discard any tasks that have lower priorities to make way for the achievement of the normative goal $[16,17]$. The Diligence function enables an agent to increase its effort in accomplishing the normative goal in time- constrained environments [16]. These two functions are used to coordinate and augment the achievement of the normative and personal goals within the duration stipulated by an authority that imposes social obligations on the society. We simulate a domain based on the OP-RND framework to show from the simulation that norms help agent cooperation in terms of accomplishing the normative goals even at the point of conflict with its other personal goals. This shows some degree of parallelism, which depends on the distribution of tasks and their concurrent execution [18].

In formalizing Sacrifice and Diligence, the architecture needs to distinguish different types of goals. We use $G_{N}$ to represent a normative goal and $G P$ to represent a personal goal. We propose two types of personal goal: mandatory and discretionary. A mandatory personal goal, GPM, is immutable and agents must mandatorily attempt to achieve the goal stipulated by all its requirements. A discretionary personal goal, GPD, is subject to changes and agents can discretionarily decide to achieve the goal, put it on hold, or reject it completely. In any domains, a deadline is one of the main influences to agents' actions and agents are penalized to resume tasks after the deadline. Consequently, agents need a flexible architecture to sacrifice one or more discretionary personal goals or to increase their diligence in 
performing tasks when imposed by some constraints of the environment. In this paper, we use agent-based simulation to compare the performance of agents in different environments and constraints. The performance is based on the sacrificial and diligence factor that leads to the achievement of some goal.

The rest of the paper is organized as follows: Section 2 discusses the related work in the research of normative agents. Section 3 proposes the normative framework in which agents interact to achieve a normative goal. Section 4 presents the case study, which is simulated in the normative framework. Section 5 presents the modeling of sacrifice and diligence functions. In Section 6, we present the results of the simulations on the agents imposed with various environmental conditions. Finally, Section 7 concludes the paper.

\section{Normative Agents}

A normative environment requires an authority that imposes some obligation and prohibition on a society in which agents as the implementers attempt to achieve a normative goal [12]. The obligation includes the tasks to be performed and their duration in the form of a start time and an end time. Under the influence of norms, a group of agents needs to observe the norms in order to fulfill the obligation or prohibition. Since norms also represent the objective of some social interactions, norms socially enforce agents' behaviours to achieve the normative goal.

Recent researches in multi-agent systems incorporate norms to influence agents' actions and improve their performance in coordination and cooperation. This claim is attested by the numerous frameworks that underpin norms to regulate the interactions in multi-agent systems $[4,7,8,11,14]$. When there are two tasks that need to be coordinated, it is important for an agent to decide which task to perform in a situation where a normative goal and personal goals co-exist. A rational agent would perform the most promising actions based on its belief of the environment. This is one of the advantages of using the mental attitudes [13] (known as belief-desire-intention) for an agent to make the most human-like decisions. Agent's beliefs, desires, and intentions have a strong relationship on executing the obligation (or prohibition) in a society. The decision-making, planning, and selection of tasks depend on the agent's behaviour towards the obligation from the authority. Figure 1 shows the diagram of agent's BDI relationship with obligation, prohibition, and norms.

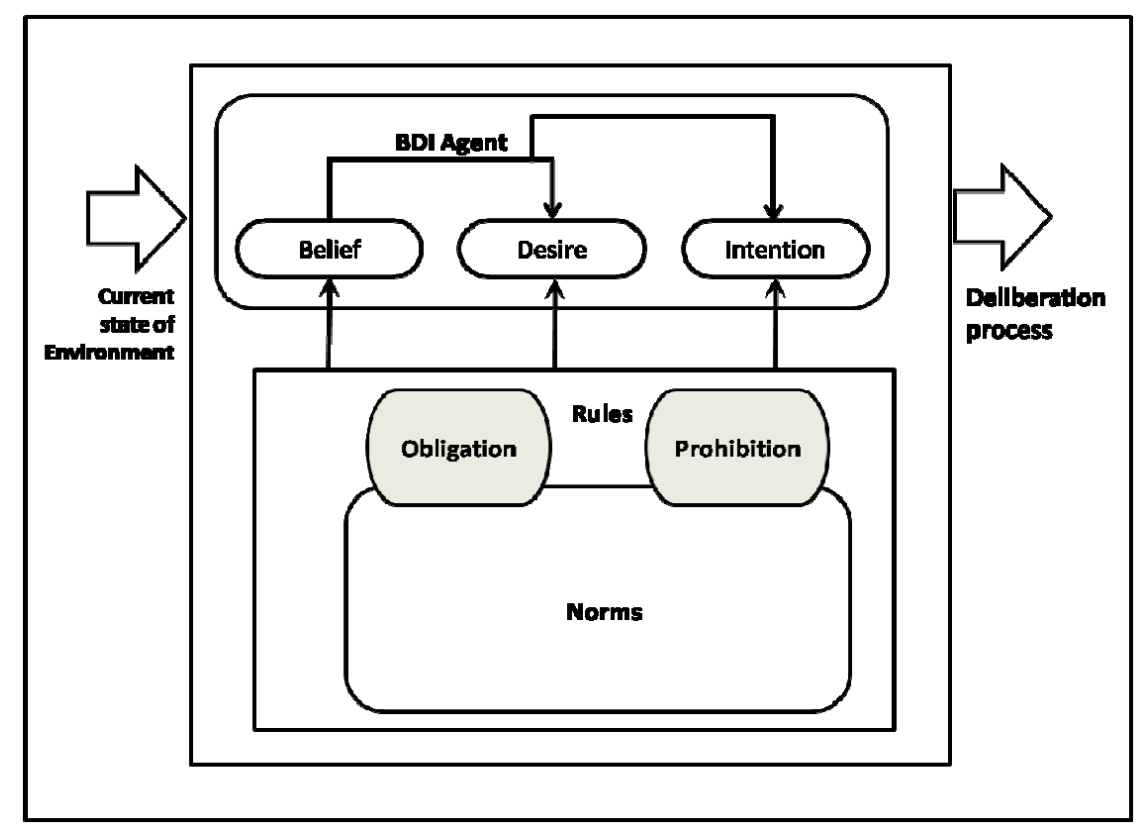

Figure 1. BDI Agent in Normative Environment

A normative autonomous agent is defined as an autonomous agent that has adopted some norms (norm instances) and has decided which norms to comply with (intended norms) and which norms to reject (rejected norms) [4]. Castelfanchi et al. [3] believe that norms are represented by mental objects entering the agents mental processes that interact with beliefs, goals and plans and a normative autonomous agent is able to take into account the existence of norms in its decision (either to follow or violate a norm) and able to react to violations of the norms by other agents. They propose deliberative normative agents that have explicit knowledge about the enacted norms in multi-agent environments and can decide whether to obey or violate the norms in specific situations. Hence, while fulfilling the obligation, agents know how and when to complete the task within the given duration.

One of the challenges in such environment is when the agent has other personal goals with deadlines. The tasks of these personal goals could conflict with the tasks of the normative goal in time, level of importance, urgency, 
durations, and other requirements that need different attention. Other researchers have attempted to resolve the issue of coordinating tasks in conflicting goals. Modgil et al. [19] use argumentation-based approach to resolve conflicts between system norms and agent's desire. The work provides agents to argue over the belief-justifying goals, conflicting preferences, and meta-level motivational argumentation over the desire, normative, penalty, and reward argued for by other agents. Thangarajah et al. [9,10] subscribe to the strategy of aborting tasks in BDI agents and a mechanism to allow agents to detect and avoid negative interactions where the effect of one goal undo conditions that are potential for another goal. Chen and Decker [20] investigate the mechanisms for agent coordination in multi-agent systems by applying coordination mechanisms for dependency relationships under various environments. They explore the specification of a large range of coordination mechanisms and the impact of task environments characteristics on the choice and performance of these mechanisms.

In this paper, we assert that the obligation comes in the form of task completion within certain duration and constraints. Similarly, in prohibition, norms enforce the agents in abstaining from performing actions that are contrary to the achievement of the normative goal. This is where sacrifice enables an agent to reason and discard any tasks that have lower priorities to make way for accomplishment of the normative goal, while diligence enables an agent to increase its effort in accomplishing the normative goal in a constrained situation.

\section{The OP-RND Framework}

In any agent actions, there are obligations, which an agent must fulfill and prohibitions, which the agent must avoid. We define Obligation, $O$, as a command imposed by some authority on the agent. In such circumstance, the agent is obligated to perform an action, gets rewarded for doing it, or penalized for leaving it. Prohibition, $P$, is defined as a command, which the agent has to avoid an action, gets rewarded for leaving it, or penalized for doing it. We consider Obligation and Prohibition (OP) as rules imposed by the authority in a normative environment due to absolute consequences (reward or penalty) upon conformation or violation of some action.

We contend that norms should exhibit weak rules or soft constraints in the environment while the agent is attempting to achieve the normative goal. We propose three principles: Recommended, Neutrality, and Disliked (RND) as norms in a normative multi-agent environment. Recommended, $R$ influences an agent in performing an action by rewarding the agent for doing it, but not penalized for leaving it. In Neutrality, $N$, the agent is not rewarded or penalized if it performs or violates an action. In Disliked, $D$, an agent is rewarded if it leaves an action, but is not penalized for doing it. However, we assert that if the agent is in the Disliked state for too many times, the state will gradually become prohibited. Consequently, we called our framework as the OP-RND Framework. We assimilate these concepts to govern the actions of agents in the proposed normative framework while maintaining the underlying semantics. In the framework, we define an agent $n$, an authority agent which imposes and monitors the rules and norms, and an agent a , the agent on which the rules and norms are imposed.

Definition 1 (Rule): A rule, $\rho$, is a mutually exclusive state of obligation, $O$ and prohibition, $P$ imposed on an agent, where:

$-O$ is a state in which the agent must perform an action and is rewarded for doing it but penalized otherwise.

$-P$ is a state in which the agent must avoid an action and is rewarded for leaving it but penalized otherwise.

Definition 2 (Norm ): A norm, $v$, is a mutually exclusive state of Recommended, $R$, Neutrality, $N$ and Disliked, $D$, where:

$-R$ is a state in which the agent is rewarded for performing an action but is not penalized otherwise.

$-N$ is a state in which the agent is neither rewarded nor penalized for performing or avoiding an action.

$-D$ is a state in which the agent is rewarded for avoiding an action but is not penalized otherwise.

Figure 2 shows an abstraction of the OP-RND framework, where Obligation and Prohibition make up the rules while Recommended, Neutrality, and Disliked constitute the norms of the framework. The outcome of the interaction within this environment influences the belief, desire, and intention of normative BDI agents. We believe that the agents should be able to perform their tasks if they could sense each state of the environment and the rewards or penalties they could get if they fulfill or violate the norms. 


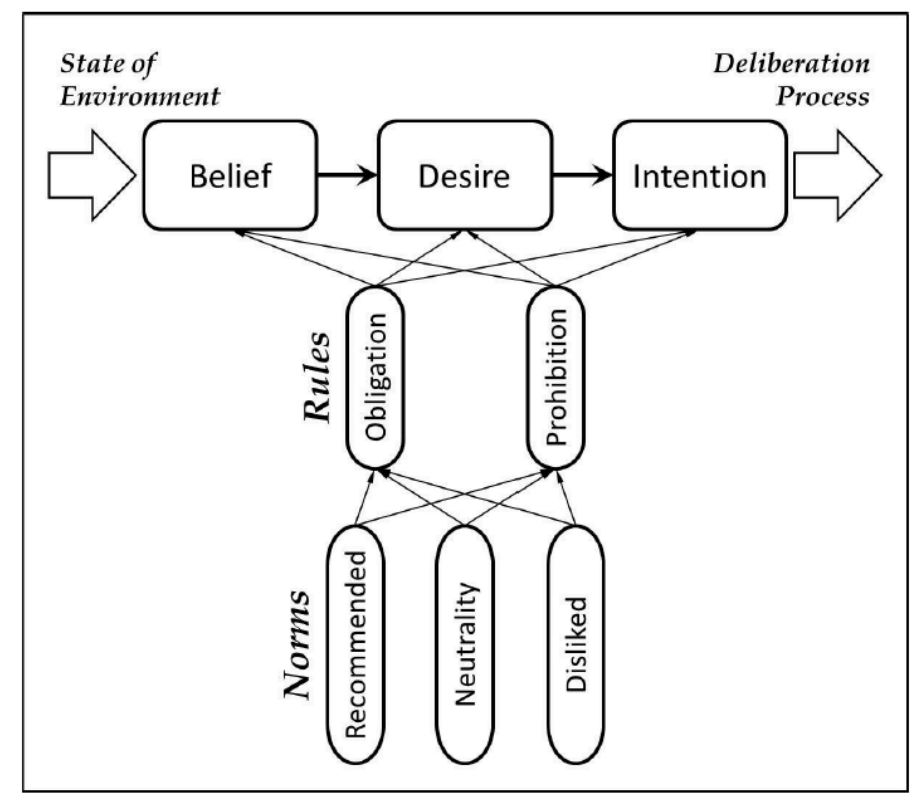

Figure 2. An abstraction of the OP-RND Framework

A definition for each of the principles is exemplified in Figure 3 for an obligation of submitting a document within a stipulated time. Given such Obligation (1), the normative influences of the Recommended (2), Neutrality (3), and Disliked (4) periods are clearly indicated in Figure 3.

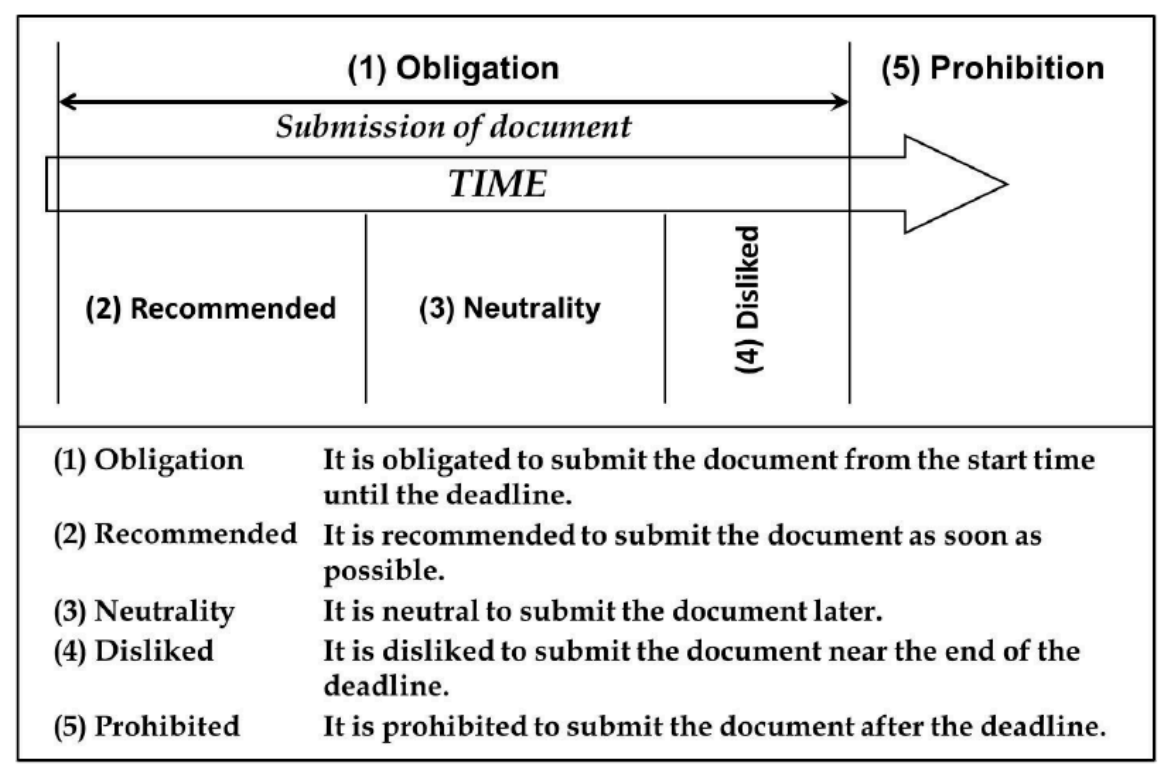

Figure 3. Semantics of the Five Principles

The figure also shows the Prohibition (5) period, which is the submission period that an agent should avoid. At the framework level, the limits between these periods are illustrated without explicit representation of any absolute values. Later, the period is quantified into number of days, $i$ and time, $\mathrm{t}$ represented as $\mathrm{t}[i]$.

Our contention in adopting these principles for a normative framework is two-fold. Firstly, some of these principles (Obligation and Prohibition) show striking similarities to the concepts proposed by other normative frameworks, [e.g. 4]. Secondly, the three selected principles (Recommended, Neutrality, and Disliked) are semantically appropriate for our model of norms, i.e., they do not exhibit the strength of rules and they have clear concepts of reward and penalty associated with them $[4,5]$.

The conception of our normative framework based on the five principles is inspired by [6]. We define a general principle $K$ in the form of $K_{x}\left(\right.$ auth,$a_{N m}$, Rew, Pen) in which an agent $a_{N m}$, being imposed by an authority agent auth, in the context $x$ of domain $s$, with reward, Rew and penalty, Pen. Agent $a_{N m}$ is the normative agent and agent auth is the agent who issues and monitors the norms. $V_{x}(s)$ is defined as the violation of $x$ by agent $a_{N m}$. We define the logical 
model of the five principles as follows:

Definition 3: Obligation, $O$ is refined as $O_{x}\left(\right.$ auth, $a_{N m}$, Rew, Pen) where agent $a_{N m}$ is obligated to agent auth to see that the obligation is in context $x$, gets rewarded with Rew or penalized with Pen, if and only if:

- $D_{\text {auth }}(x) \vee G_{\text {auth }}(x) \rightarrow G_{a N m}(x)$ : If the context is $x$, then agent auth desires and has a goal $x$, and this goal is distributed to agent $a_{N m}$.

- $\neg x \rightarrow V_{x}(s)$ : If $\neg x$, then agent auth has the goal and the desire $V_{x}(s)$; to recognize it as a violation by agent $a_{N m}$.

- $V_{x}(s) \rightarrow$ Pen: Agent auth decides $V_{x}(s)$, then agent auth desires and has a goal that penalizes agent $a_{N m}$.

- $\neg V_{x}(s) \rightarrow$ Rew: If there is no violation, then agent auth has the goal and the desire to reward agent $a_{N m}$.

Definition 4: Prohibition, $P$ is refined as $P_{x}\left(\right.$ auth, $a_{N m}$, Rew, Pen) where agent $a_{N m}$ is obligated to agent auth to see that the prohibition is in context $x$, gets rewarded with Rew or penalized with $P$ en, if and only if:

- $D_{\text {auth }}(x) \vee G_{\text {auth }}(x) \rightarrow G_{a N m}(x)$ : If the context is $x$, then agent auth desires and has a goal $x$, and this goal is distributed to agent $a_{N m}$.

- $\neg x \rightarrow V_{x}(s)$ : If $\neg x$, then agent auth has the goal and the desire $V_{x}(s)$; to recognize it as a violation by agent $a_{N m}$.

- $V_{x}(s) \rightarrow$ Pen: Agent auth decides $V_{x}(s)$, then agent auth desires and has a goal that penalizes agent $a_{N m}$.

- $\neg V_{x}(s) \rightarrow$ Rew: If there is no violation, then agent auth has the goal and the desire to reward agent $a_{N m}$.

Definition 5: Recommended, $R$ is refined as $R_{x}\left(\right.$ auth, $a_{N m}$, Rew, Pen) where agent $a_{N m}$ is obligated to agent auth to see that the recommended is in context $x$, gets rewarded with Rew or penalized with Pen, if and only if:

- $D_{\text {auth }}(x) \vee G_{\text {auth }}(x) \rightarrow G_{a N m}(x)$ : If the context is $x$, then agent auth desires and has a goal $x$, and this goal is distributed to agent $a_{N m}$.

- $\neg x \rightarrow \neg V_{x}(s)$ : If $\neg x$, then agent auth has the goal and the desire $V_{x}(s)$; to recognize it as a non-violation by agent aNm.

- $V_{x}(s) \rightarrow \neg$ Pen: Agent auth decides $V_{x}(s)$, then agent auth desires and has a goal that does not penalizes agent $a_{N m}$.

- $\neg V_{x}(s) \rightarrow R e w:$ If there is no violation, then agent auth has the goal and the desire to reward agent $a_{N m}$.

Example 1: There is an agent $L$, who needs to submit a document at time $t$ (i.e., week 10), and submits it at an earlier week, such as week 8 . Each of the examples shown below corresponds to the above model:

a) Submit_document $\rightarrow\{$ submit, $t\}$

b) Submit_document $\rightarrow\{\neg$ submit, $t\}$

c) $\left\{\right.$ Submit_document, $V\left(\right.$ submit, $\left.\left.a_{N m}\right)\right\} \rightarrow \neg$ Pen

d) $\left\{\right.$ Submit_document, $\neg V\left(\right.$ submit,$\left.\left.a_{N m}\right)\right\} \rightarrow$ Rew

In this example, agent $L$ is the agent $a_{N m}$ who needs to follow the norms and agent auth monitors the norm. Based on Figure 3, submission of document at an earlier time is recommended and will be rewarded by agent auth (line (d). It is not a violation if agent $a_{N m}$ does not perform the submission (line (b) and (c)) and will not be penalized.

Definition 6: Neutrality, $N$ is refined as $N_{x}\left(\right.$ auth, aNm, Rew, Pen) where agent $a_{N m}$ is obligated to agent auth to see that neutrality is in context $x$, gets rewarded with Rew or penalized with Pen, if and only if:

- $D_{\text {auth }}(x) \vee G_{\text {auth }}(x) \rightarrow G_{a N m}(x)$ : If the context is $x$, then agent auth desires and has a goal $x$, and this goal is distributed to agent $a_{N m}$.

- $\neg x \rightarrow V_{x}(s)$ : If $\neg x$, then agent auth has the goal and the desire $V_{x}(s)$; to recognize it as a non-violation by agent aNm.

- $V_{x}(s) \rightarrow \neg$ Pen, $\neg$ Rew: Agent auth decides $V_{x}(s)$, then agent auth desires and has a goal that does not penalizes agent $a_{N m}$.

- $\neg V_{x}(s) \rightarrow \neg$ Rew, $\neg$ Pen: If there is no violation, then agent auth has the goal and the desire not to reward agent $a_{N m}$.

Example 2: There is an agent $L$, who needs to submit a document at time $t$ (i.e., week 10), and submits the document on week 10.

a) Submit_document $\rightarrow\{$ submit, $t\}$

b) Submit_document $\rightarrow\{\neg$ submit, $t\}$

c) $\left\{\right.$ Submit_document, V(submit, $\left.\left.a_{N m}\right)\right\} \rightarrow \neg P e n$

d) $\left\{\right.$ Submit_document, $\neg V\left(\right.$ submit,$\left.\left.a_{N m}\right)\right\} \rightarrow \neg$ Rew

Referring to Figure 3, submission of document on time is neutral and is not rewarded or penalized if agent $a_{N m}$ complies or violates the rule (line (c) and (d)). 
Definition 7: Disliked, $D$ is refined as $D_{x}\left(a u t h, a_{N m}\right.$, Rew, Pen) where agent $a_{N m}$ is obligated to agent auth to see that disliked is in context $x$, gets rewarded with Rew or penalized with Pen, if and only if:

- $D_{\text {auth }}(x) \vee G_{\text {auth }}(x) \rightarrow G_{a N m}(x)$ : If the context is $x$, then agent auth desires and has a goal $x$, and this goal is distributed to agent $a_{N m}$.

- $\neg x \rightarrow V_{x}(s)$ : If $\neg x$, then agent auth has the goal and the desire $V_{x}(s)$; to recognize it as a non-violation by agent $a_{\text {Nm. }}$

- $V_{x}(s) \rightarrow \neg P e n$ : If there is no violation, then agent auth has the goal that does not penalize agent $a_{N m}$.

- If $N$ represents the number of times agent $a$ complete the task in Disliked state and $N>3 ; V_{x}(s, N) \rightarrow P e n$ : if $x$ and agent auth decides $V_{x}(s, N)$, then it is recognized as a violation by agent auth, then agent auth desires and has a goal that penalizes agent $a_{N m}$.

Example 3: There is an agent $L$, who needs to submit a document at time $t$ (i.e., week 10), but does not submit it until week 13 (i.e. before the deadline on week 14).

a) Submit_document $囚\{$ submit, $t\}$

b) Submit_document $\{\neg$ submit, $t\}$

c) $\left\{\right.$ Submit_document, $V\left(\right.$ submit,$\left.\left.a_{N m}\right)\right\} \rightarrow$ Rew

d) $\left\{\right.$ Submit_document, $\neg V\left(\right.$ submit,$\left.\left.a_{N m}\right)\right\} \rightarrow \neg P e n$

e) $\left\{\right.$ Submit_document, $\neg V\left(\right.$ submit $\left.\left., a_{N m}, N\right)\right\} \rightarrow$ Pen

In Figure 3, submission of document at a much later time is disliked and is rewarded if agent $a_{N m}$ violates the rule (line (c)). But if agent $a_{N m}$ does it many times, it will become a violation by agent auth. In these three examples, as illustrated in Figure 3, we show that agent $a_{N m}$ is aware of the normative states (i.e., $R$, $N$, and $D$ ) of the environment. The reward and penalty models in each state influence the agents to decide accordingly.

As mentioned earlier, we model the framework on rules and norms. The rules define specific conditions of obligation and prohibition which are mutually exclusive and influence the execution of norms within the community. In the framework, a rational agent exercises its norms within the duration of its obligation and attempt to avoid encroachment into the prohibited area. While the agent is at liberty to violate the norms (e.g., exercising its norms in the Disliked state), the framework's reward/penalty structure motivates the agent to execute its actions in more favorable states, i.e., Recommended or Neutrality. The side effect of these outcomes manifests a significant improvement in agent coordination and subsequently improves the overall performance.

\section{Case Study}

We select the domain of examination paper preparation and moderation process (EPMP) of our College as the case for our study. In the EPMP, a Lecturer is obligated to prepare and submit a complete examination paper set (questions, solutions, and marking scheme) within two weeks to a Moderator who reviews the paper. However, within the same duration, the Lecturer is also responsible to other goals that need to be accomplished along with the paper preparation and submission tasks. In this instance, we call the paper submission to the Moderator as the normative goal, $G_{N}$, which needs to be fulfilled. The other goals, which we called personal goals, $G_{P}$, are based on the need of each individual Lecturer, such as taking leave, attending workshops or courses, attending meeting or supervising students. Both $G_{N}$ and $G_{P}$ need to be scheduled during the two weeks duration.

We simulate the above scenario in the OP-RND framework, which espouses five principles: Obligation, Prohibition, Recommended, Neutrality, and Disliked as the normative framework for multi-agent systems [13]. For the simulation, we use 14 days to represent the obligation period. If $E n v_{N}$ is the normative environment, $P_{E}$ is the evaluated points (reward, none, or penalty), and $\delta_{S}$ is the document's submission date, then the agent's normative belief, $B_{N}$, of its reward or penalty is as follows:

$$
B_{N}: \operatorname{brf}\left(E n v_{N} \cup P_{E}\right) \rightarrow \delta s
$$

where $\delta_{S}=\mathrm{t}[i]$, and $i=1 \ldots 14 . P_{E}$ is a candidate of the agent's belief. We rewrite the above formula as:

$$
B_{N}: \operatorname{brf}\left(E n v^{\prime}\right) \rightarrow \delta s, \text { where } E n v^{\prime}=E n v_{N} \cup P_{E}
$$

The expression means that the agent's normative belief is its belief revision function (brf) of the environment and the evaluated points, which implies the document's submission date.

The agent receives a reward, none, or penalty for submission under the three normative periods based on the following conditions:

- Reward, when $\mathrm{t}[5] \geq \delta_{S} \geq \mathrm{t}[1]-$ Recommended,

- None, when $\mathrm{t}[12] \geq \delta_{S} \geq \mathrm{t}[6]-$ Neutral,

- Penalty, when $\mathrm{t}[14] \geq \delta_{S} \geq \mathrm{t}[13]$ - Disliked.

The agent's desire $D$, is represented by the tasks which the agent could perform based on its belief. We model the agent's desire with a set of pre-compiled tasks, $T$, which the agent could select. Based on the EPMP, 
the agent could perform any of the following pre-compiled tasks, $\tau_{i} \in T$, depending of the current state of the environment and its belief:

- $\tau_{1}$ : Submit documents, i.e., when all recommended slots, $\mathrm{t}[1]$ to $\mathrm{t}[5]$ have $G_{N}$ values.

- $\tau_{2}$ : Submit documents after a sacrifice is invoked, i.e., when only some slots have $G_{P D}$.

- $\tau_{3}$ : Submit documents after a diligence is invoked, i.e., when only some slots have $G_{P M}$.

- $\tau_{4}$ : Submit documents after sacrifice and diligence are invoked, i.e., when some slots have $G_{P D}$ and $G_{P M}$.

The tasks which the agent selected based on its belief and desire represent its intention, $I$. It then commits resources to complete the tasks, which lead to the achievement of its normative goal. We model the selection of tasks by the agent or its intention as a filter function of the state of the environment, Env', its evaluation of the reward or penalty, $P_{E}$, and the set of tasks, $T$, i.e.:

$$
I: \operatorname{filter}\left(E n v^{\prime}, T\right) \rightarrow \tau_{i} \text {, where } i^{3} 1 \text {. }
$$

\section{Modeling Sacrifice and Diligence Functions}

In handling multiple goals for normative multi-agent environments, the OP-RND framework is designed to regulate rules and norms effectively. The framework is used as the platform to handle multiple goals in timeconstrained situations. We introduce two additional functions to the BDI architecture of the OP-RND framework: Sacrifice and Diligence. These two functions are used to optimize the performance of normative and personal tasks within the duration stipulated by the authority.

The Sacrifice function is defined as follows, where $T_{\mathrm{XS}}, \mathrm{T}_{\mathrm{XE}}$ are the start $(\mathrm{S})$ and end $(\mathrm{E})$ times of the normative periods (X), namely, the Recommended, Neutrality, and Disliked periods:

- The agent believes on the normative goal

- The agent believes that the conditions for achieving the normative goal in the Recommended period, $\mathrm{T}_{\mathrm{RE}^{-}}$ $\mathrm{T}_{\mathrm{RS}}$, have become false.

- The agent believes that discretionary personal goal can be cancelled or postponed.

- The agent believes on getting the reward in the Recommended period, $T_{R E}-T_{R S}$.

- The agent believes on being penalized in the Disliked period, $\mathrm{T}_{\mathrm{DE}}-\mathrm{T}_{\mathrm{DS}}$.

- The agent believes on not getting reward in the Neutrality period, $\mathrm{T}_{\mathrm{NE}}-\mathrm{T}_{\mathrm{NS}}$.

- The agent desires on getting the reward in the Recommended period, $T_{R E}-T_{R S}$.

- The agent desires on avoiding the penalty in the Disliked period, $\mathrm{T}_{\mathrm{DE}}-\mathrm{T}_{\mathrm{DS}}$.

If $S$ is the Sacrifice function then,

$$
S: \operatorname{brf}\left(\operatorname{Env}_{N}, P_{E}, G_{P D}, G_{N}\right) \rightarrow I
$$

i.e., the agent performs the sacrifice function based on the environment, $E n v_{N}$, the evaluated points, $P_{E}$, and the number of $G_{P D}$ and $G_{N}$, which implies the agent's intention, $I$.

The Diligence function is defined as follows:

- The agent believes on the normative goal.

- The agent believes that the conditions for achieving the normative goal in the Recommended period, $\mathrm{T}_{\mathrm{RE}^{-}}$ $\mathrm{T}_{\mathrm{RS}}$, have become false.

- The agent believes that the mandatory personal goal cannot be cancelled or postponed.

- The agent believes on increasing its effort on the task in the Recommended period, $T_{R E}-T_{R S}$.

- The agent believes on getting the reward in the Recommended period, $T_{R E}-T_{R S}$.

- The agent believes on being penalized in the Disliked period, $\mathrm{T}_{\mathrm{DE}}-\mathrm{T}_{\mathrm{DS}}$.

- The agent believes on not getting reward in the Neutrality period, $\mathrm{T}_{\mathrm{NE}}-\mathrm{T}_{\mathrm{NS}}$.

- The agent desires on getting the reward in the Recommended period, $T_{R E}-T_{R S}$.

- The agent desires on avoiding the penalty in the Disliked period, $\mathrm{T}_{\mathrm{DE}}-\mathrm{T}_{\mathrm{DS}}$.

We model the Diligence function to enable the agent to apply the function only if there are many $G_{P M}$ tasks slotted within a normative period and the agent believes that it would not be able to submit within that period, i.e., if $\delta_{S}>\mathrm{t}[5]$ for the Recommended period. If $D_{X}$ is the diligence function under a normative period $\mathrm{X}$, then,

$$
D_{X}: \operatorname{brf}\left(E n \operatorname{En}_{N}, P_{E}, G P M\right) \rightarrow I
$$

i.e., the agent performs the Diligence function based on the environment, $E n v_{N}$, the evaluated points, $P_{E}$, and the number of $G_{P M}$, which implies the agent's intention, $I$. The degree of agent's diligence depends on the period in which submission is made. We model the degree of diligence at the Recommended $\left(D_{\mathrm{R}}\right)$, Neutral $\left(D_{\mathrm{N}}\right)$, and Disliked $\left(D_{\mathrm{D}}\right)$ periods as follows:

- $\quad$ Recommended, $D_{\mathrm{R}}=100 /\left(5-G^{P M}\right)$, when $5 \geq G_{P M} \geq 1$.

- $\quad$ Neutral, $D_{\mathrm{N}}=100 /\left(12-G_{P M}\right)$, when $12 \geq G_{P M} \geq 6$.

- $\quad$ Disliked, $D_{\mathrm{D}}=100 /\left(14-G_{P M}\right)$, when $14 \geq G_{P M} \geq 13$.

When Diligence is invoked, the agent increases its effort to perform the task quicker to get the reward and avoid the 
penalty. We assume that it is possible for the agent to complete the task in one slot.

We redefine the agent's intention to include the Sacrifice and Diligence functions as follows:

$$
I: \text { filter }\left(T, S, D_{X}\right) \rightarrow \tau_{i} \text {, where } i \geq 1 \text {. }
$$

i.e., the expression means that the agent's intention is a function of the set of tasks, and the result of the sacrifice and diligence functions. This implies a selected task, $\tau_{i}$ from $\mathrm{T}$.

\section{Simulation and Analysis of Results}

We simulate the EPMP domain using 100 agents on all simulations based on the OP-RND framework and the following percentage of goal distributions imposed of the EPMP stakeholders resulted from a survey [2]: $G_{N}=$ $10 \%, G_{P D}=30 \%$, and $G_{P M}=60 \%$.

This percentage represents real occurrences of multiple goals as surveyed on the Lecturers in the EPMP domain. The normative goal, $G_{N}$, is the submission of the moderated EPMP documents to the Examination Committee. The personal discretionary goals, $G_{P D}$ include On Leave/Sick Leave/Not Feeling Well, Emergency Leave (e.g. car broke down), Bank/Post Office/Government Agencies, Attend Wedding/Family Matters and Attend Sick Children/Parents/Relatives. Finally, the personal mandatory goals, $G_{P M}$ include Administration, Teaching Preparation, Supervision, Research, Publication, and Study (Master/PhD). form.

In this research, we simulate the following five modes of environments and present the results in graphical

- $\quad$ Mode A - Normative BDI with single goal, $G_{N}$.

- $\quad$ Mode B - BDI only with multiple goals, $G_{N}, G_{P M}$, and $G_{P D}$.

- Mode C - BDI and Sacrifice function with multiple goals, $G_{N}, G_{P M}$, and $G_{P D}$.

- Mode D - BDI and Diligence function with multiple goals, $G_{N}, G_{P M}$, and $G_{P D}$.

- Mode E - BDI with Sacrifice and Diligence functions, and with multiple goals, $G_{N}, G_{P M}$, and $G_{P D}$.

\subsection{Mode A - BDI only with single goal, $G_{N}$}

Figure 4 shows the simulation results for the BDI with a single goal, $G_{N}$. The figure shows the distribution of submissions between $\mathrm{t}[1]$ to $\mathrm{t}[15]$. The figure shows an expected submission spike at $\mathrm{t}[5]$ due to the absence of other goals, GPD or GPM during the obligation period. The results indicate that without any constraints, agents are able to comply with the norms.

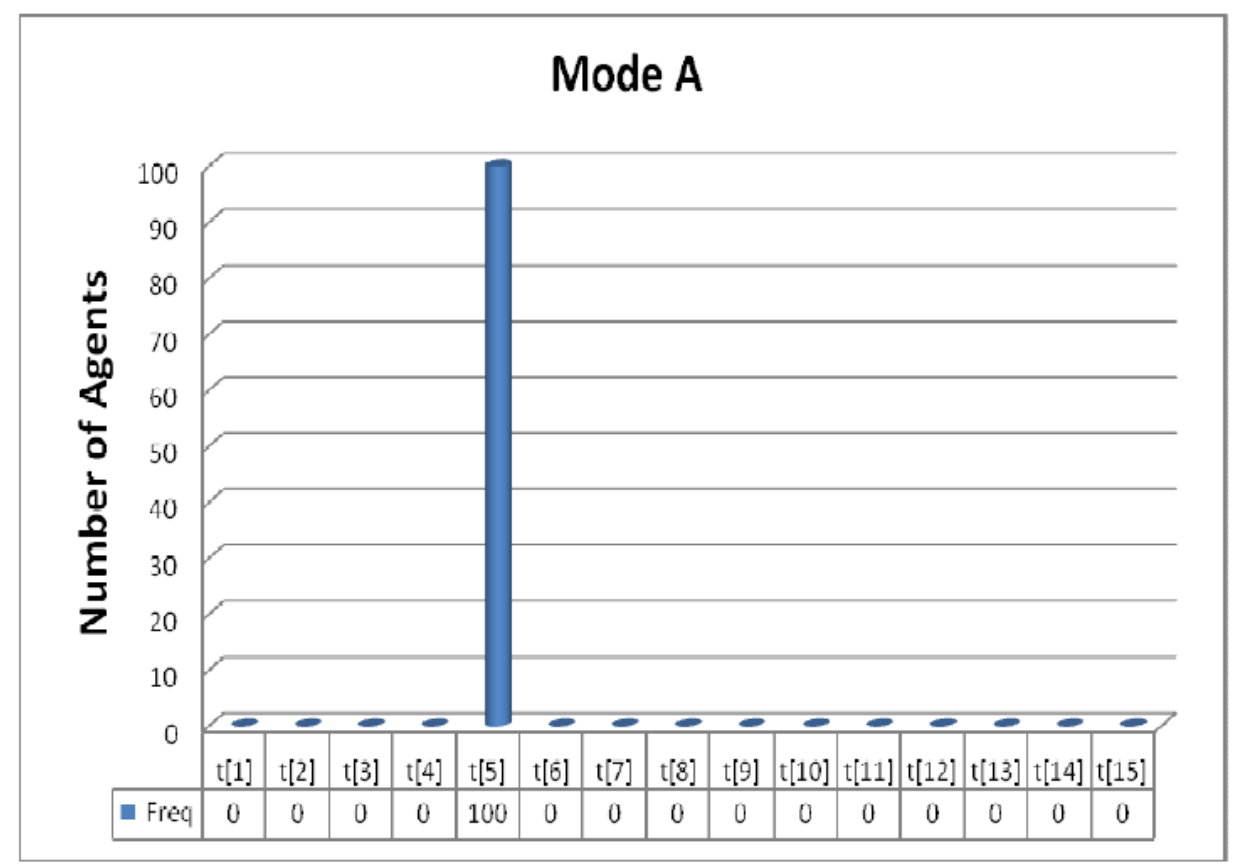

Figure 4. Simulation with BDI and Single Goal 


\subsection{Mode B - BDI only with multiple goals, $G_{N}, G_{P M}$, and $G_{P D}$}

Figure 5 shows the simulation results for the BDI with multiple goals.

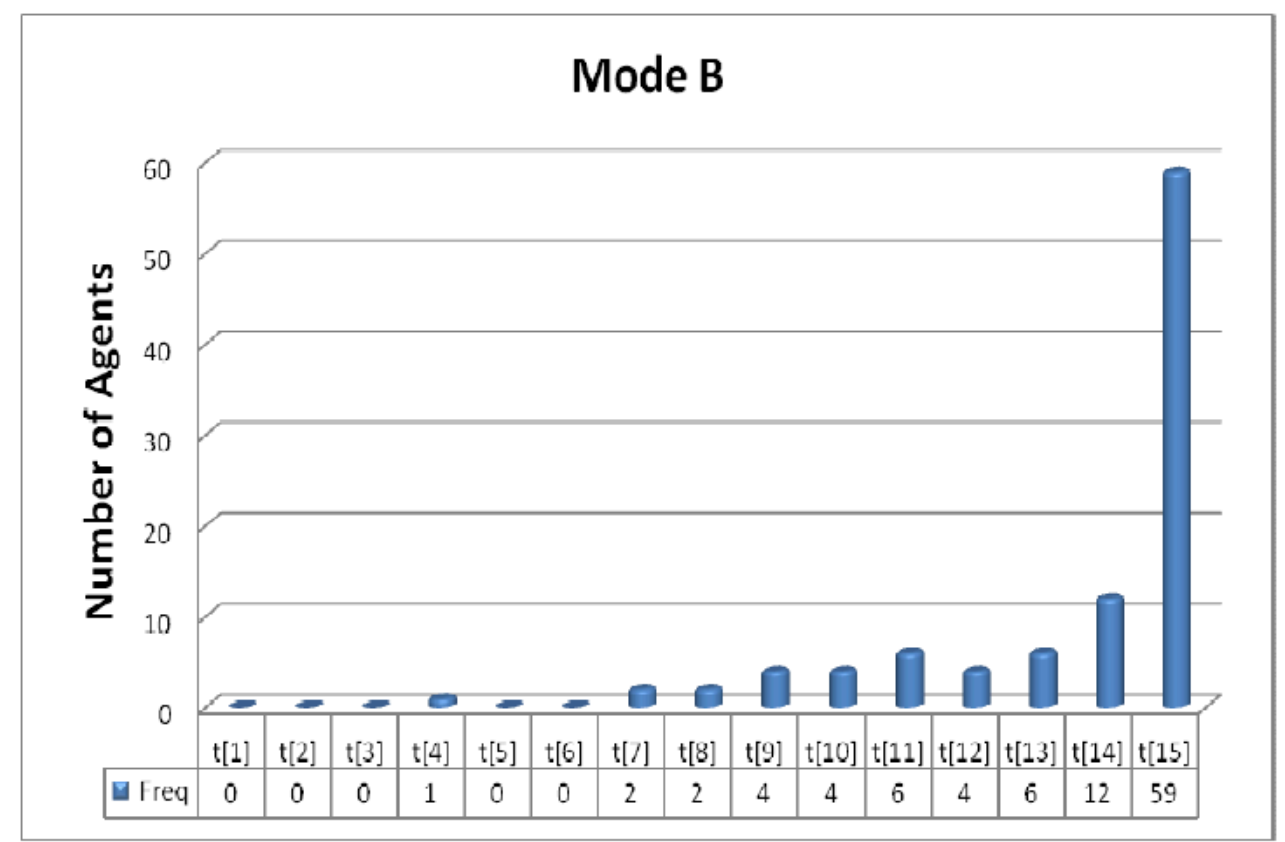

Figure 5. Simulation with BDI and Multiple goals

The figure shows the distribution of submissions between $t[1]$ to $t[15]$. About 18 agents submitted in the Disliked period at $\mathrm{t}[13]$ and $\mathrm{t}[14]$ and more than 50 agents submitted in the Prohibited period. All other agents submitted in the Neutral period and none submitted in the Recommended period. The results indicate that a majority of agents are unable to comply with the norms. Without sacrifice and diligence and with $G_{P D}$ and $G_{P M}$, the agents are unable to submit within the Recommended period

\subsection{Mode C - BDI with Sacrifice function with multiple goals, $G_{N}, G_{P M}$, and $G_{P D}$}

Figure 6 shows the results of simulation with the Sacrifice function. About three agents managed to submit within the Recommended period. Many other agents submitted in the Neutrality period, about ten agents submitted in the Disliked period and 34 agents submitted in the Prohibition period. The results indicate a somewhat improved performance in norms compliance but still a large number of agents are unable to submit within the Recommended or Neutrality period. Clearly, with the Sacrifice function alone, not many agents are able to submit the documents within the Recommended period, which could be due to many $G_{P M}(60 \%)$ in that period.

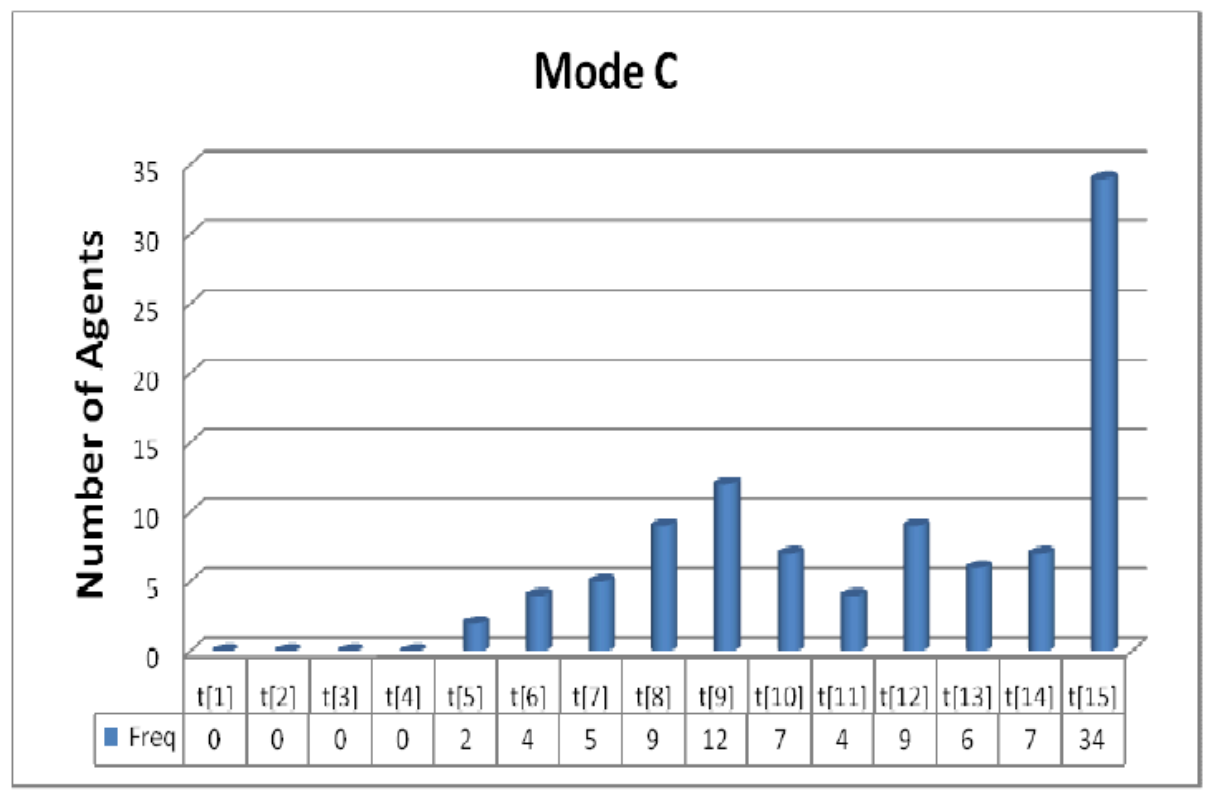

Figure 6. Simulation with BDI and Sacrifice function only 


\subsection{Mode D - BDI with Diligence function with multiple goals, $G_{N}, G_{P M}$, and $G_{P D}$}

Figure 7 shows the results of simulation with the Diligence function. The results indicate that the performance of the agents is much better because agents' diligence is increased due to many $G_{P M}$. About 64 agents managed to submit within the Recommended period. The rest submitted in the Neutrality period and none submitted in the Disliked and Prohibited period. The results indicate a significant improvement in the agents' compliance with the norms

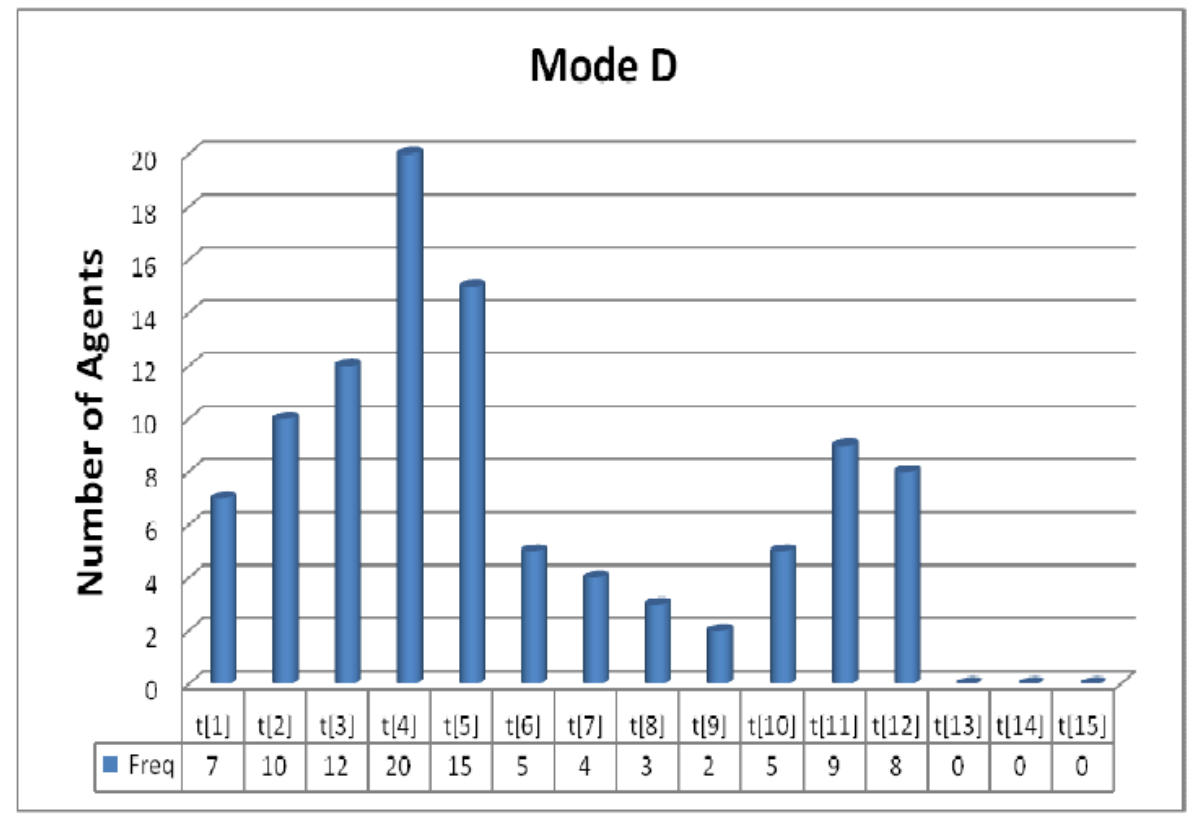

Figure 7. Simulation with BDI and Diligence function only

\subsection{Mode E - BDI with Sacrifice and Diligence function, and with multiple goals, $G_{N}, G_{P M}$, and $G_{P D}$}

Figure 8 shows the results of simulation with the Sacrifice and Diligence functions. The results show the distribution of submissions between $\mathrm{t}[1]$ and $\mathrm{t}[15]$, indicating that the agents' performance is very much improved. All agents (99\%) managed to submit the documents within the Recommended and Neutrality period.

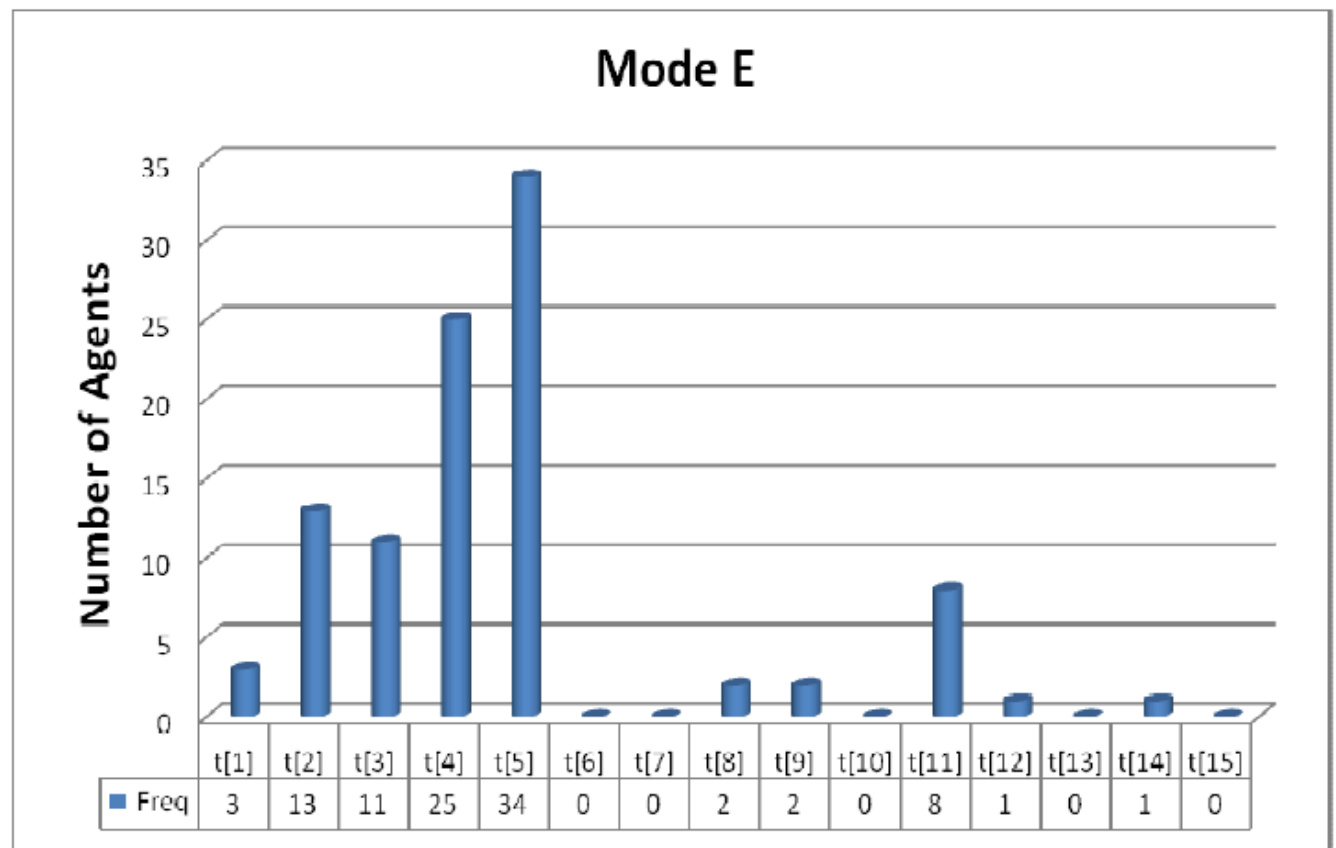

Figure 8. Simulation with BDI and Sacrifice and Diligence functions 


\subsection{Comparison on all models}

We compare the agent performance in each mode by computing the mean, median, mode, and standard deviation of the submissions. Table 1 shows such comparison

Table 1 Statistics of Mode A to Mode E

\begin{tabular}{|c|c|c|c|c|c|}
\hline & Mode A & Mode B & Mode C & Mode D & Mode E \\
\hline Mean & 5.00 & 13.55 & 11.64 & 5.63 & 4.80 \\
\hline Median & 5.00 & 15.00 & 12.00 & 5.00 & 4.00 \\
\hline Mode & 5 & 15 & 15 & 4 & 5 \\
\hline $\begin{array}{c}\text { Std. } \\
\text { Deviation }\end{array}$ & .000 & 2.354 & 3.183 & 3.410 & 2.655 \\
\hline
\end{tabular}

From the table, we assert that Mode E, where agents implement the Sacrifice and Diligence functions under our normative framework, indicates the submission at a statistical mode of 5 and an average time of 4.8 , which is in the Recommended period. Agents are aware on the normative period and the reward in the Recommended period and thus, able to submit early in this environment.

If we compare with Mode D where agents only use the Diligence function, we can see the difference due to their inability to invoke the Sacrifice function to postpone or cancel the discretionary goals, $G_{P D}$, if any. The small difference is due to the $60 \%$ occurrences of $G_{P M}$, where agents increase their diligence for all cases. The other modes, which are Mode A, Mode B and Mode C, show a clear case of late submissions and norm violations in each of these environments. The Sacrifice and Diligence function enables the early submissions of the document hence improves the agents' performance. Therefore, we assert that our normative framework shows better performance with the Sacrifice and Diligence functions, thus giving a positive impact on the submission time.

Figure 9 illustrates the complete model of the EPMP operating under the OP-RND framework with the Sacrifice and Diligence functions. Such architecture could resolve the problems of multiple goals achievement in time-constrained situations.

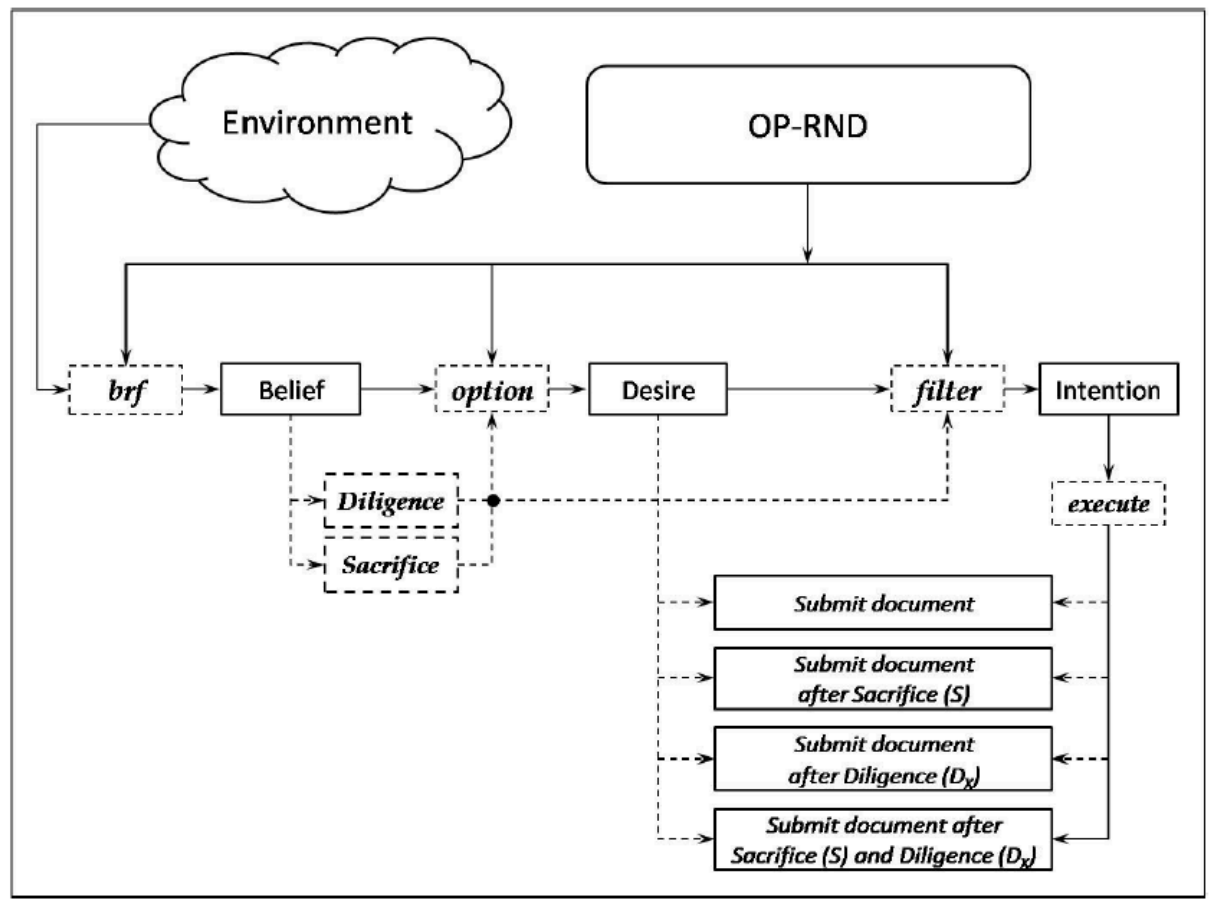

Figure 9. The Normative BDI Architecture with Sacrifice and Diligence

\section{Conclusions}

In this paper, we presented a framework for conflict resolution of multiple goals in a normative environment. We introduced the Sacrifice and Diligence functions in the OP-RND normative framework. Such 
functions are required to provide a robust architecture for multi-agent systems in which agents are imposed with multiple goals. The need to comply with the norms in a time-constrained environment of multiple goals requires an architecture that enables agents to sacrifice their personal goals and the ability to increase its effort in completing their tasks earlier.

The simulations for all environments are based on the actual situation of multiple goals occurrences in real life, which are derived from a survey. From the survey, the mandatory goals occurred the most during the obligation period. This situation is common among humans and they often resort to similar actions when faced with overwhelming constraints. We model the architecture based on such situation and simulate the model on our normative framework on five different modes. The results clearly show that the BDI architecture with Sacrifice and Diligence functions work well for agents working in time-constrained multiple goals' environments while at the same time maintaining compliance with the norms.

In our future work, we will investigate two issues: the need to model "increase in diligence" of the agents in time constrained cases and to characterize the personal goals, i.e., to study the circumstances under which the goals are mandatory or discretionary, or having variable durations, because in real situations, such goals have their own characteristics and demand different attention.

\section{References}

[1] A. Ahmad, M.S. Ahmad, M.Z.M. Yusoff, and A. Mustapha, A Novel Framework for Normative Agent-Based Systems, MJCAI 2009, Kuala Lumpur, Malaysia, 2009.

[2] A. Ahmad, M. Ahmed, M.Z.M. Yusoff, M.S. Ahmad, A. Mustapha, Resolving Conflicts between Personal and Normative Goals in Normative Agent Systems, The Seventh International Conference on IT in Asia 2011 (CITA 2011), pp. 153 - 158, ISBN: $983-$ 9257-66-8, Kuching, Sarawak, 12 - 14 July 2011.

[3] C. Castelfranchi, F. Dignum, C.M. Jonker, and J. Treur, Deliberative Normative Agents : Principles And Architecture, 6th International Workshop on Intelligent Agents VI, Agent Theories, Architecture and Languages (ATAL09), Orlando, Florida, 1999.

[4] F.L.y. Lopez, and A.A. Marquez, An Architecture for Autonomous Normative Agents, 5th Mexican International Conference on Computer Science (ENC 2004), IEEE Computer Society 2004, ISBN 0-7695-2160-6, Colima Mexico, 2004.

[5] F.S.A. Majeed, Islamic Law (al-Fiqh), Our Printer Pte Ltd, Singapore, 1990

[6] G. Boella, and L.v.d. Torre, Regulative and Constitutive Norms in Normative Multi-agent Systems, In Procs. of 9th International Conference on the Principles of Knowledge Representation and Reasoning. Whistler (KR04), Whistler, Canada, 2004.

[7] G. Boella, and L.v.d. Torre, Fulfilling or Violating Obligations in Normative Multi-agent System, IAT 2004, Beijing, China, 2004, pp. 483-486.

[8] G. Boella, and L.v.d. Torre, Norm Governed Multi-agent System: The Delegation of Control to Autonomous Agents, IAT 2003 Halifax, Canada, 2003, pp. 329-335.

[9] J. Thangarajah, J. Harland, D. Morley, N. Yorke-Smith, 2007. Aborting Tasks in BDI Agents, AAMAS 2007, Hawaii.

[10] J. Thangarajah, L. Padgham, M. Winikoff, 2003. Detecting \& Avoiding Interference between Goals in Intelligent Agents, IJCAI'03: Proceedings of the 18th International Joint Conference on Artificial Intelligence, Morgan Kaufmann Publishers Inc, pp. 721-726.

[11] L. Astefanaoei, M. Dastani, J.-J. Meyer, and F.S.d. Boer, A Verification Framework for Normative Multi-Agent Systems, PRIMA 2008, Hanoi, Vietnam, 2008.

[12] Lacroix, P. Mathieu, and A. Kemeny, The Use of Norms' Violation to Model Agents' Behavioral Variety, AAMAS $2008,2008$.

[13] M. J. Wooldridge, N. R. Jennings, 1995. Intelligent Agents: Theory and Practice, Knowledge Engineering Review, 10(2).

[14] M. Kollingbaum, I.J. Jureta, W. Vasconcelos, and K. Sycara, Automated Requirements-Driven Definition of Norms for the Regulation of Behavior in Multi-agent System, AISB2008, Aberdeen, Scotland, 2008.

[15] M. Neumann, Norm Internalisation in Human and Artificial Intelligence. Journal of Artificial Societies and Social Simulation JASSS 13 (1) 12 (2010)

[16] M.Z.M. Yusoff, M. Ahmed, A. Ahmad, M.S. Ahmad, and A. Mustapha, Sacrifice and Diligence in Normative Agent Environments, The 2010 International Conference on Computer and Software Modeling (ICCSM 2010), Phillipines, 2010.

[17] M.Z.M. Yusoff, Y. Khalid, A. Ahmad, M.S. Ahmad, and A. Mustapha, Development of Rules for Multiple Goals in Normative Environments, MJCAI2010, Sarawak, Malaysia, 2010.

[18] R.R. Dumke, R. Koeppe, and C. Wille, Software Agent Measurement and Self-Measuring Agent-Based Systems, Technical Report II, University of Madgeburg, 2000.

[19] S. Modgil, and M. Luck, Argumentation based Resolution of Conflicts Between Desires and Normative Goals. LNCS 2009 (2009) 19-36.

[20] W. Chen, and K. Decker, Applying coordination Mechanisms for Dependency Relationships under Various Environments, Multiagent Systems, Artificial Societies, and Simulated Organizations, Springer US, 2004. 